This item was submitted to Loughborough's Research Repository by the author.

Items in Figshare are protected by copyright, with all rights reserved, unless otherwise indicated.

\title{
The effect of collagen ageing on its structure and cellular behaviour
}

PLEASE CITE THE PUBLISHED VERSION

https://doi.org/10.1117/12.908749

PUBLISHER

(C) Society of Photo-Optical Instrumentation Engineers (SPIE)

VERSION

VoR (Version of Record)

LICENCE

CC BY-NC-ND 4.0

REPOSITORY RECORD

Wilson, Samantha L., Marie Guilbert, Josep Sule-Suso, James Torbet, Pierre Jeannesson, Ganesh D.

Sockalingum, and Ying Yang. 2019. "The Effect of Collagen Ageing on Its Structure and Cellular Behaviour". figshare. https://hdl.handle.net/2134/34609. 


\section{The effect of collagen ageing on its structure and cellular behaviour}

Samantha L. Wilson, Marie Guilbert, Josep Sulé-Suso, James Torbet, Pierre Jeannesson, et al.

Samantha L. Wilson, Marie Guilbert, Josep Sulé-Suso, James Torbet, Pierre Jeannesson, Ganesh D. Sockalingum, Ying Yang, "The effect of collagen ageing on its structure and cellular behaviour," Proc. SPIE 8222, Dynamics and Fluctuations in Biomedical Photonics IX, 822210 (9 February 2012); doi: 10.1117/12.908749

SPIE. Event: SPIE BiOS, 2012, San Francisco, California, United States 


\title{
The effect of collagen ageing on its structure and cellular behaviour
}

\author{
Samantha L. Wilson ${ }^{\mathrm{a}}$, Marie Guilbert ${ }^{\mathrm{b}}$, Josep Sulé-Suso ${ }^{\mathrm{a}, \mathrm{c}}$, James Torbet ${ }^{\mathrm{d}}$, Pierre Jeannesson ${ }^{\mathrm{b}}$, \\ Ganesh D. Sockalingum ${ }^{\mathrm{b}}$ and Ying Yang. ${ }^{\mathrm{a}}$ \\ ${ }^{a}$ ISTM, Keele University, Stoke-on-Trent, ST4 7QB, UK \\ ${ }^{\mathrm{b}}$ Equipe MéDIAN, CNRS UMR 6237, UFR de Pharmacie, Université de Reims \\ Champagne-Ardenne, 51 rue Cognacq-Jay, 51096 Reims, France \\ ${ }^{c}$ Cancer Centre, University Hospital of North Staffordshire, Stoke on Trent, ST4 6QG, UK \\ ${ }^{\mathrm{d}}$ The High Magnetic Field Laboratory, LNCMI, 38042 Grenoble, France
}

\begin{abstract}
Collagen is the most important component in extracellular matrix (ECM) and plays a pivotal role in individual tissue function in mammals. During ageing, collagen structure changes, which can detrimentally affect its biophysical and biomechanical properties due to an accumulation of advanced glycation end-products (AGEs). AGEs have been linked to non-enzymatic cross-linking of proteins resulting in the alteration of mechanical properties of the tissue. In this study we investigate the influence of different aged collagens on the mechanical and contractile properties of reconstituted hydrogel constructs seeded with corneal stromal fibroblasts. A non-destructive indentation technique and optical coherence tomography (OCT) are used to determine the elastic modulus and dimensional changes respectively. It is revealed that the youngest collagen constructs have a higher elastic modulus and increased contraction compared to the older collagen. These results provide new insights into the relationship between collagen molecular structures and their biomechanical properties.
\end{abstract}

Keywords: collagen ageing, Advanced Glycation End-products, cell contraction, elastic modulus.

\section{INTRODUCTION}

Collagen is a key life-long structural protein that constitutes approximately one-third of the total mass in the body. ${ }^{1}$ Understanding the properties and functions of collagen will aid our understanding of physiological and pathophysiological situations, and should be useful when manipulating and constructing in vitro tissues. Collagen fibrils consist of tropocollagen triple helices, with cross-links between and within the triple helices ${ }^{2}$ (Fig. 1). Detrimental changes to biochemical and biomechanical interactions are all a result, amongst other causes, of the ageing process ${ }^{3,4}$ and consequently collagen loses flexibility, enzymatic digestibility ${ }^{5}$ and becomes less deformable. ${ }^{6,7}$ Collagen has active reactive groups and any chemical alterations to collagen molecules after synthesis will have a direct effect on tissue and organ function. ${ }^{5,6,8}$ Such changes are often associated with inter- and intra-molecular cross-linking of the peptides. Crosslinking can occur enzymatically (lysyloxidase mediated ${ }^{6}$ ) or non-enzymatically. Enzymatic processes usually occur during developmental and maturation stages of the collagen; whereas non-enzymatic changes occur due to the ageing process and are damaging to the protein and associated biomolecules. ${ }^{5}$ Frequently non-enzymatic changes are a result of glycation reactions caused by the accumulation of advanced glycation end-products (AGEs).

AGEs are a complex and heterogeneous group of compounds whose presence have been shown to increase in tissues with increasing age. ${ }^{7}$ The exact mechanisms that lead to AGE formation still elude us. What is known is that AGE formation causes proteins to cross-link which is the underlying process by which they cause damage. AGE accumulation in collagen is the result of a reaction of the oxo-group of sugars with a free $\varepsilon$-amino group of the protein (amino group of lysine or arginine). ${ }^{6}$ The initial Schiff base (a compound with a functional group containing a carbon-

*Corresponding author: Email:y.yang@bemp.keele.ac.uk; Telephone: +44 (0)1782 554606

Dynamics and Fluctuations in Biomedical Photonics IX, edited by Valery V. Tuchin,

Donald D. Duncan, Kirill V. Larin, Martin J. Leahy, Ruikang K. Wang, Proc. of SPIE Vol. 8222,

$822210 \cdot$ (C) 2012 SPIE · CCC code: 1605-7422/12/\$18 - doi: 10.1117/12.908749

Proc. of SPIE Vol. 8222 822210-1 
nitrogen double bond, with nitrogen connected to an aryl or alkyl group) and resulting Amadori products undergo an irreversible series of reactions resulting in cross-linked structures ${ }^{5}$ (Fig. 1). Cross-linking interferes in cell-matrix interactions affecting adhesion and cell spreading function and decreasing tissue remodelling capabilities. ${ }^{7}$ Such changes are accelerated by some physiological situations such as ageing and pathophysiological conditions such as diabetes. 9 The formation of AGE is catalysed by transitional metals and inhibited by reducing compounds such as ascorbate. In vitro studies have also shown that insulin removes AGEs. ${ }^{7}$ The assessment of non-enzymatic changes is difficult ${ }^{5}$ and unfortunately AGE measurement varies widely between studies. Many different methods exist for measuring AGE and as of yet there is no gold standard method used to detect AGE. However, the methods most commonly used include HPLC, ELISA and immunohistochemistry. ${ }^{7}$

In this study we aim to investigate the effect of collagen structural changes due to ageing on their mechanical properties and capacity of cellular contraction in reconstituted collagen hydrogel constructs by a non-destructive indentation technique ${ }^{12}$ and imaging modality. We hypothesise that inter- and intramolecular cross-linking of collagen interferes greatly with the fibrillogenesis process and consequently, the mechanical properties of the hydrogel construct will be affected. Furthermore, non-destructive imaging techniques ${ }^{12}$ can provide direct and indirect evidence for this structure-property relationship of aged collagens.

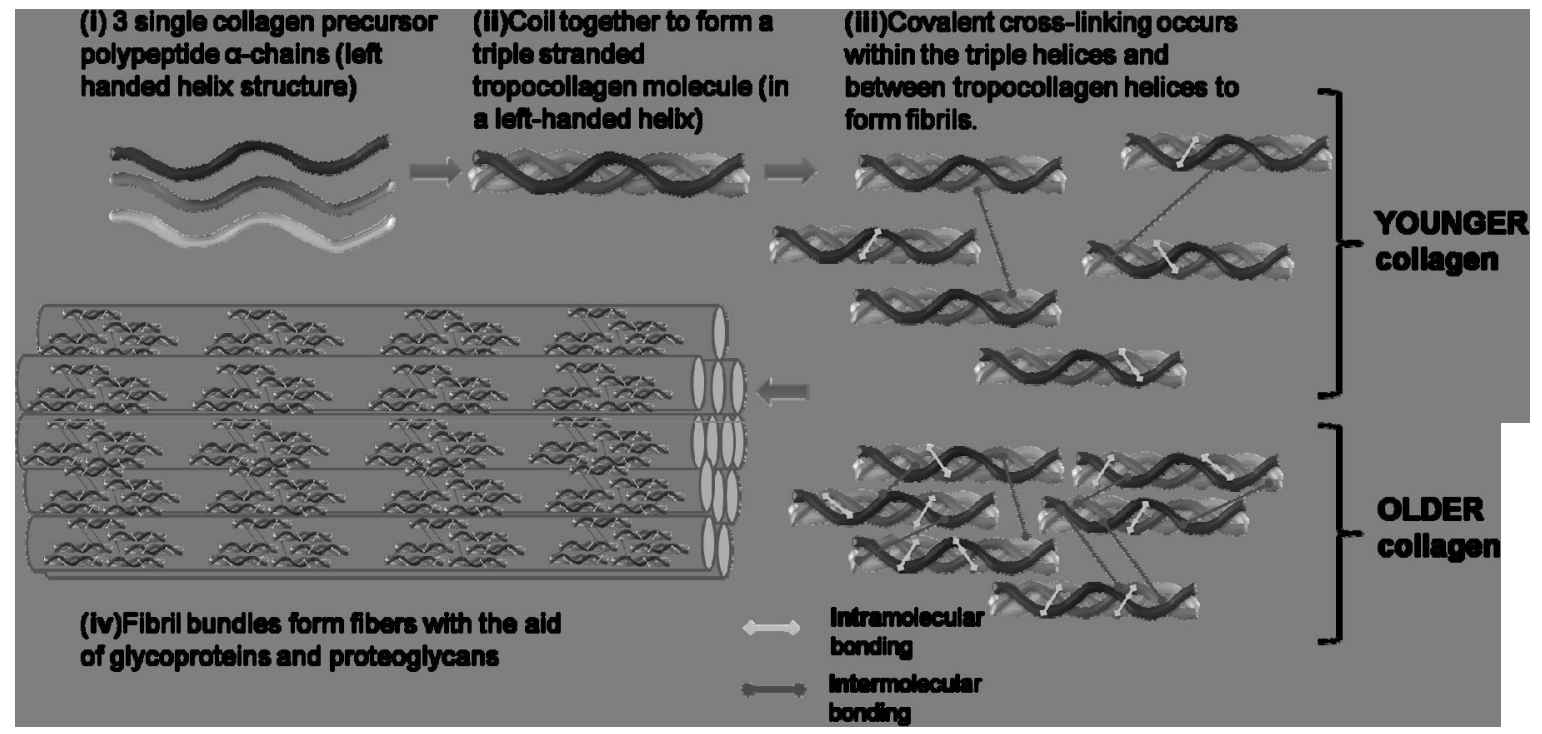

Figure 1: Collagen structure. Accumulation of AGEs with ageing causes an increase in intra and intermolecular collagen cross-linking which results in an increase in collagen density.

\section{MATERIALS AND METHODS}

\subsection{Corneal Stromal Cell Culture}

Human corneal tissue remaining from corneal transplantation was used for the isolation of stromal cells. This research has received approval from Birmingham NHS Health Authority Local Research Ethics Committee. The endothelial and epithelial layers were physically stripped using sharp-point forceps. The remaining stromal layer was cut into smaller pieces and cultured in cell culture flasks containing Dulbecco's modified eagle medium (DMEM; Biowest, France) supplemented with $10 \%$ foetal calf serum (FCS, Biowest, France), $1 \%$ antibiotic and antimycotic solution (A+A, Sigma-Aldrich, UK) and $2 \mathrm{mM} \mathrm{L-Glutamine} \mathrm{(Sigma-Aldrich,} \mathrm{UK)} \mathrm{at} 37^{\circ} \mathrm{C}, 5 \% \mathrm{CO}_{2}$, allowing stromal cells to migrate out from the tissue. Media was changed every 2-3 days and the cells were passaged before reaching confluence. Third passage corneal stromal cells were used for these experiments. 


\subsection{Collagen Extraction}

Native type I collagen from rat tail tendons at different ages, 2-4 days (baby), 8 weeks, 24 weeks and 96 weeks was extracted as previously described. ${ }^{10,11}$ Briefly, acid-soluble type I collagen was obtained from rat tail tendons by acetic acid extraction then purified by dialysis against distilled water and lyophilised. This lyophilised, purified, and nonpepsined collagen was stored at $-80^{\circ} \mathrm{C}$ until further use. For 3D gels preparation, lyophilised collagens were sterilised in ethanol, dried and then dissolved in sterile $18 \mathrm{mM}$ acetic acid at a concentration of $5 \mathrm{mg} / \mathrm{ml}$.

\subsection{Fabrication of Acelular and Cell-containing Hydrogels}

To neutralize the collagen solution and to fabricate collagen hydrogel constructs, $\mathrm{NaOH}$ and other cellsupporting additives were added to the given collagen solution. In order to make $1 \mathrm{ml}$ of neutralized collagen gel solution, $100 \mu \mathrm{l}$ MEM (10X concentration), $100 \mu \mathrm{l} \mathrm{NaHCO}_{3}(22 \mathrm{~g} / \mathrm{l}), 90 \mu \mathrm{l} 0.1 \mathrm{M} \mathrm{NaOH}, 10 \mu \mathrm{l}$ L-Glutamine (100X concentration), $100 \mu \mathrm{l} \mathrm{H}_{2} \mathrm{O}, 100 \mu \mathrm{l}$ FCS and $500 \mu \mathrm{l}$ collagen at concentration $5 \mathrm{mg} / \mathrm{ml}$ were mixed. $500 \mu \mathrm{L}$ collagen mixture of final concentration $2.5 \mathrm{mg} / \mathrm{ml}$ were cast into a filter paper ring (internal diameter $20 \mathrm{~mm}$ ) on non-adherent PTFE plates. Gelation was achieved by incubation at $37^{\circ} \mathrm{C}, 5 \% \mathrm{CO}_{2}$ for $45-60$ mins. The cell-containing hydrogels were formed following the same protocol as the acellular hydrogel except that $0.5 \times 10^{6}$ cells per construct were suspended in $100 \mu \mathrm{L}$ FCS. The cells were suspended throughout the hydrogel solution prior to gelation at a concentration of $1 \times 10^{6}$ cells $/ \mathrm{ml} .500 \mu \mathrm{L}$ of the cell-collagen mixture were cast for each construct.

\subsection{Gel Contraction}

Hydrogels cast into filter paper rings were effectively confined to the dimension of the ring. This allowed the analysis of confined contraction in terms of a change in thickness of the hydrogel construct via optical coherence tomography (OCT, see section 2.7). Acellular collagen hydrogels were produced as a control. Three specimens per group have been tested. Recorded data was averaged and represented as a mean value \pm the calculated standard deviation.

\subsection{Cell Viability}

Cell viability in the constructs was evaluated at day 7 using a live-dead double staining kit (Fluka, Switzerland) able to simultaneously stain viable and dead cells. The kit contained two solutions, Calcein-AM (solution A, green) and Propidium Iodide (solution B, red). $500 \mu \mathrm{l}$ of the staining solution was used for each construct. The hydrogel constructs were then examined using fluorescent microscopy (Eclipse Ti-S, Nikon, Japan).

\subsection{Modulus Measurement}

The mechanical properties of the constructs were measured daily by the indentation technique ${ }^{12}$ after they were prepared. The instrumentation consists of a sample holder with a spherical indenter and an image acquisition system. The technique involves indenting a circumferentially clamped hydrogel construct using a sphere of known weight and radius and measuring the deformation caused centrally to the construct. The central deformation displacement is applied to a theoretical mode ${ }^{13}$ to calculate the elastic modulus of the hydrogels. The non-destructive nature of the technique allows repeated measurement of individual constructs over prolonged culture periods.

OCT was used to measure the change in thickness of constructs daily. The detailed description of the instrument can be found elsewhere. ${ }^{14}$ The acquired images were analysed using ImageJ (NIH, USA). Recorded data was averaged and represented as a mean value \pm the calculated standard deviation. Acellular control data was relatively constant for the duration of the experiment so the data was accumulated and displayed as an average of 7 days and represented as a mean value \pm the calculated standard deviation.

\subsection{Magnetic Alignment of Collagen}

Acellular collagen hydrogel samples were prepared as described previously (see section 2.3.) at concentration $1.5 \mathrm{mg} / \mathrm{ml}$. The collagens were aligned under 12 Tesla magnetic fields (CRETA, Grenoble) during fibrillogenesis. The magnetically aligned hydrogels were viewed using polarised light microscopy (SP-60P, Brunel, UK) and the collagen fibre organisation images were captured using digital photography (D5000, Nikon, Japan). 


\section{RESULTS}

\subsection{Gel Contraction in Cellular Constructs}

Differences in viscosity were observed between the four collagen groups. The older collagen solutions (24 and 96 week) were visibly more viscous than the younger collagen (babies and 8 week) at the same concentration. Although all collagens formed homogenous hydrogels, the gelation time for the younger collagen was longer compared to the older collagen. The corneal fibroblasts in the hydrogel caused contraction during the culture. However, the contraction of the constructs was limited to a change in thickness since the presence of the filter paper ring prevented the hydrogels from contracting horizontally. The change in thickness of the constructs was measured using OCT daily for 7 days (Fig. 2). The thickness of all acellular constructs remained constant throughout the duration of the experiment. All cellular constructs contracted thus making the constructs thinner. Most contraction occurred within the first 3 days. ANOVA tests revealed that the age of the collagen had a statistically significant effect on the change in thickness of the construct. The youngest collagen, 2-4 days (baby) showed the greatest degree of contraction and by the end of the experiment was less than $20 \%$ of its original thickness (Fig. 4). The baby collagen decreased significantly more in thickness than all other ages of collagen used $(p \leq 0.001$ days 1-7 inclusive for age 8, 24 and 96, although the statistical significance was less for days 6-7 in 8 week collagen where $\mathrm{p}<0.05$ ). Similar trends were observed when comparing 8 week collagen to 24 and 96 week collagen ( $\leq 0.001$ days 1-7 inclusive). 24 week collagen also decreased in thickness significantly more than 96 week collagen ( $\mathrm{p} \leq 0.05$ day $2-4 ; \mathrm{p} \leq 0.01$ day $3-5 ; \mathrm{p} \leq 0.001$ day $6-7$ ).

\subsection{Modulus Measurement}

The elastic modulus of the different aged collagen constructs in both acellular and cellular constructs was measured daily for 7 days. All acellular control hydrogels retained a constant modulus with a visible trend for the younger the collagen, the higher the modulus (Fig. $\mathbf{2}$ and $\mathbf{3}$ ). The modulus of the baby constructs was significantly greater than 24 week and 96 week constructs for the duration of the experiment ( $\mathrm{p} \leq 0.001$ inclusive). However the baby collagen only showed a significantly greater modulus compared to 8 week collagen at day $4(\mathrm{p} \leq 0.05)$ and day 6 (p $\leq$ 0.01 ). Similar trends were observed when comparing the modulus of 8 week collagen to 24- and 96 week collagen ( $\mathrm{p} \leq$ 0.001 day 2-7 inclusively but statistically less significant for 24 week collagen at day 5 where $p \leq 0.05$ ). 24 week collagen also had a significantly higher modulus compared to 96 week collagen ( $p \leq 0.05$ day 2 and $4 ; p \leq 0.01$ day 3 and $5 ; \mathrm{p} \leq 0.001$ day 6-7). 

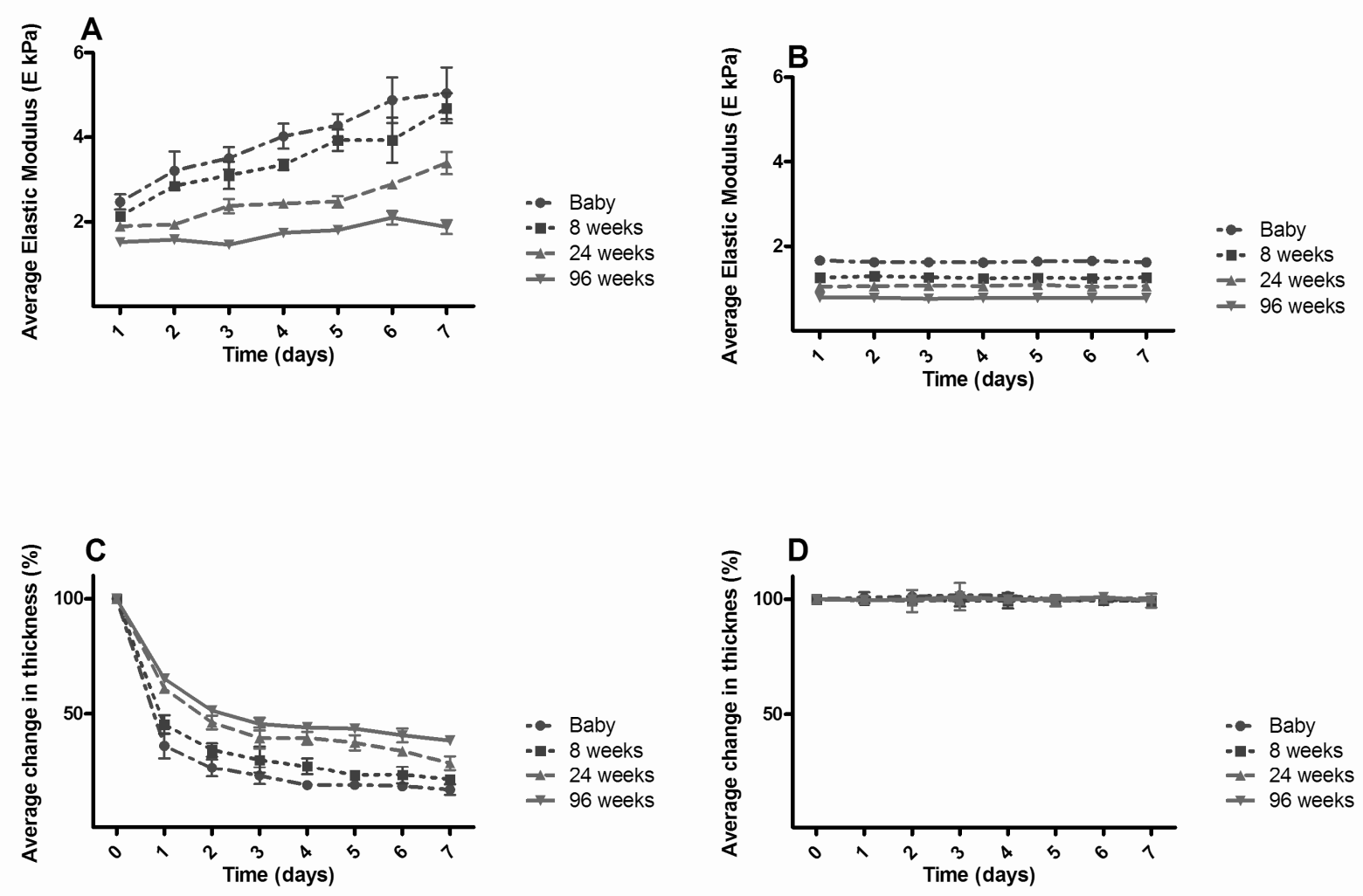

Figure 2: The average elastic modulus of confined cellular (A) and acellular (B) constructs using collagen extracted from different aged rats cultured for 7 days; is directly linked to the average change in thickness of the cellular (C) and acellular (D) constructs. $n=3$.

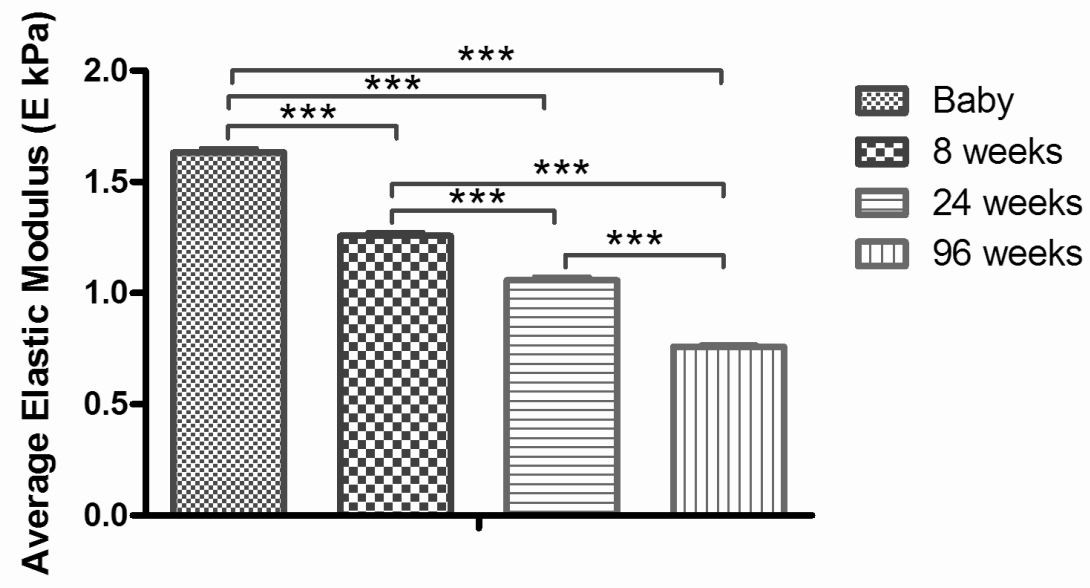

Figure 3: Average elastic modulus from 7 days of culture in acellular constructs using collagen extracted from different aged rats. $\mathrm{n}=3, * * *=\mathrm{p} \leq 0.001$. 


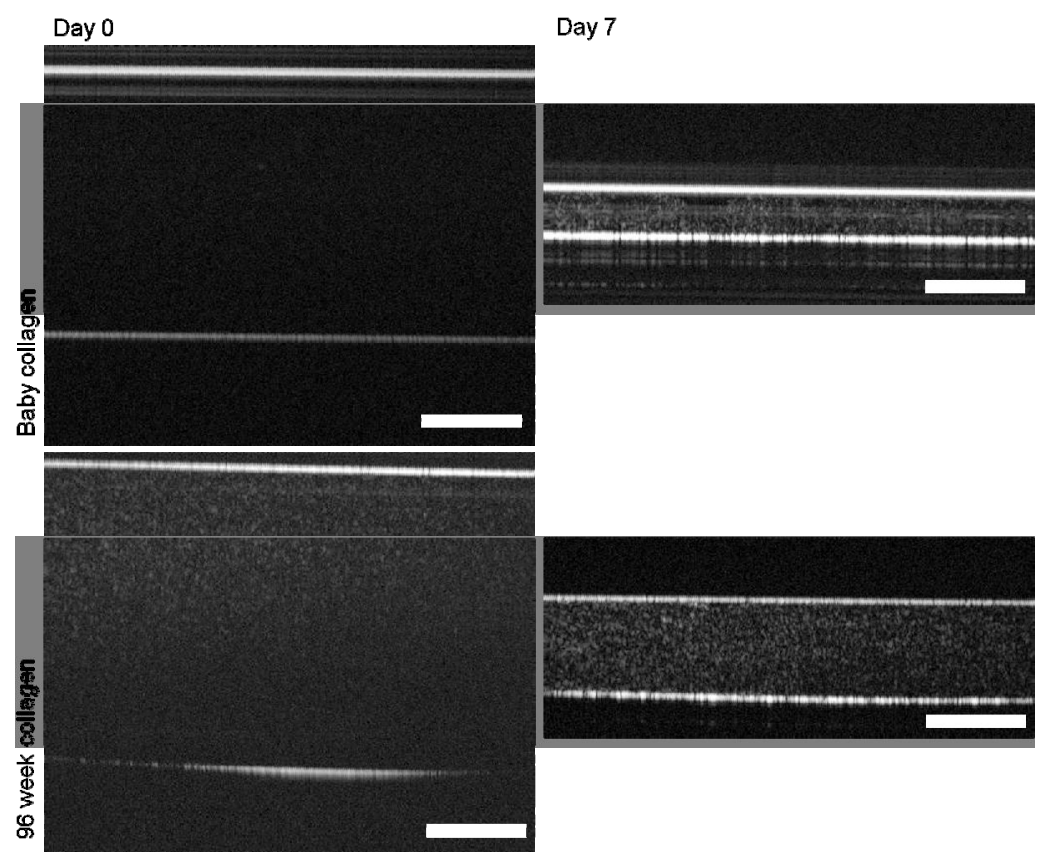

Figure 4: Comparative OCT images of cellular collagen constructs manufactured using baby and 96 week old collagen at day 0 and 7. Both cell-seeded constructs contract, but the degree of contraction is significantly less in the older collagen specimens. Scale bar $=500 \mu \mathrm{m}$.

\subsection{Cell Viability}

All constructs had good cell viability following 7 days culture (Fig. 5) with no obvious differences in cell viability under different ages of collagen.
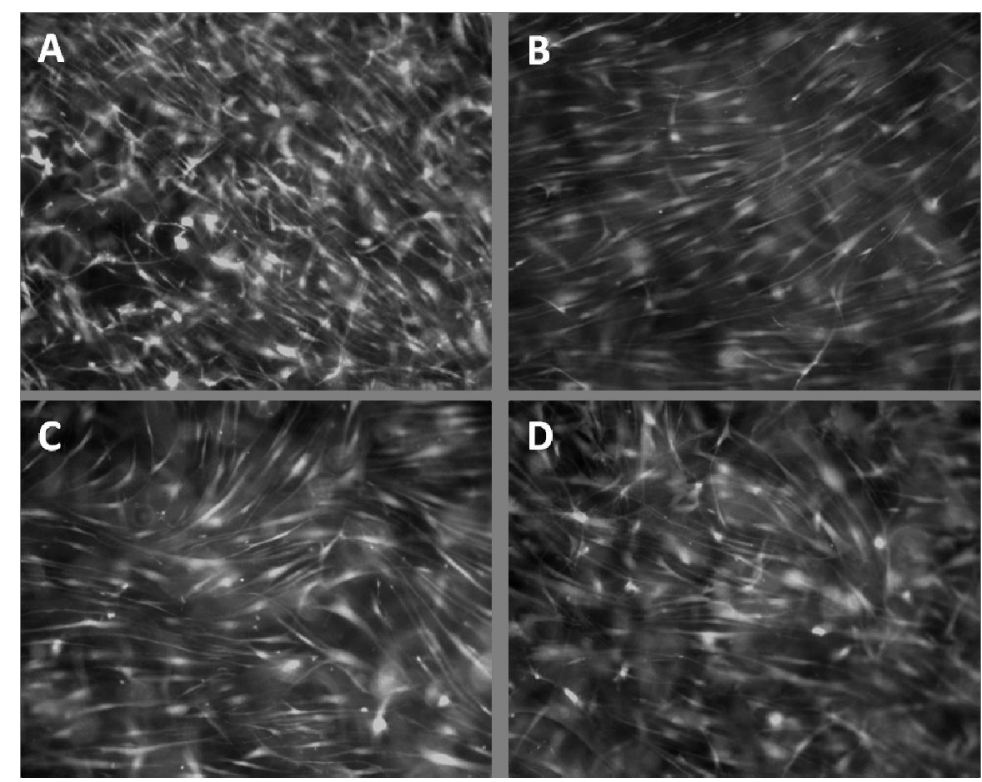

Figure 5: Representative live-dead images of collagen gels from rats aged 2-4 days (A); 8 weeks (B); 24 weeks (C); and 96 weeks (D) following 7 days culture. 


\subsection{Magnetic alignment}

Following exposure to a 12 Telsa magnetic field during fibrillogenesis with a step heating programme, collagen fibres in 8 week samples aligned perpendicular to the applied magnetic filed, whereas fibres in the older (96 week) and baby specimens remained random in orientation (Fig. 6).
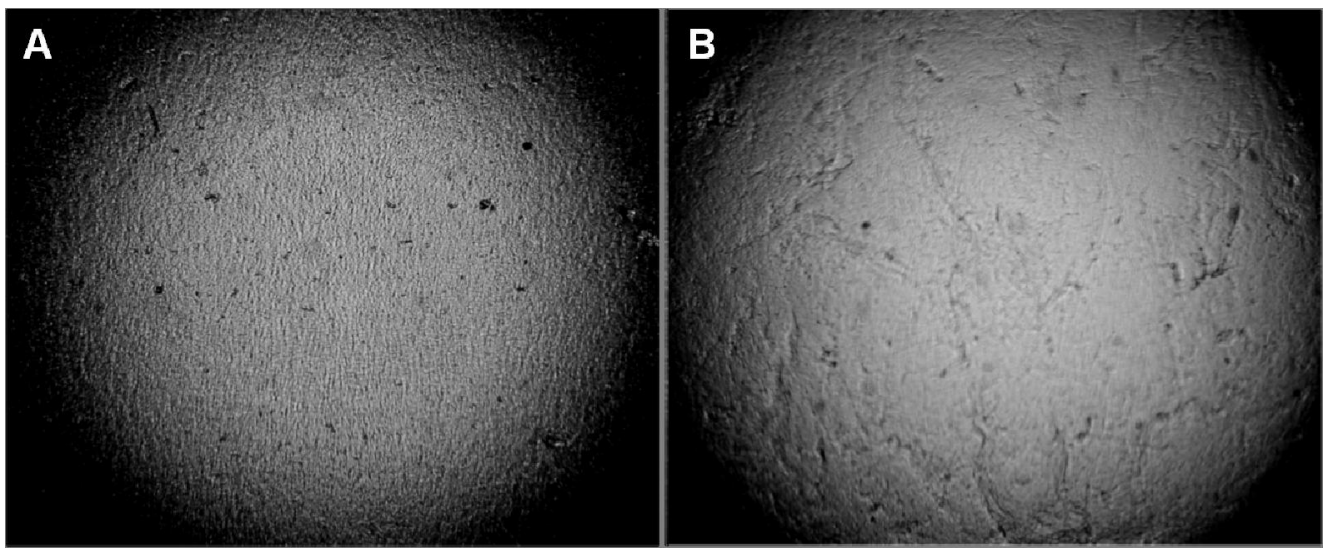

Figure 6: Representative Polarised light microscope images of 8 week (A) and 96-week (B) collagen samples fibrillogenesis under $12 \mathrm{~T}$ magnetic field.

\section{DISCUSSION}

Collagen type I is a fibre-forming protein and an important scaffolding component in the ECM. Therefore, the quality and quantity of collagen monomers (tropocollagen), fibril diameter and fibre bundle diameter and morphology all directly influence fibrillogenesis and the mechanical properties of the resulting hydrogel construct. In our study, through measurement of the macroscopic parameters including observation of collagen monomer viscosity, fibril alignment measurement of elastic modulus (acellular hydrogel) and the contraction capability (thickness change and elastic modulus), we have obtained further evidence and new insights that ageing changes the collagen monomer structure. These changes considerably affect the fibrillogenesis process, which alters the architecture of collagen fibres in the hydrogel.

The higher viscosity of the older collagen solutions (before gelation) indicated that a high population of the collagen molecules were not in the tropocollagen form and that the corresponding molecular weight of collagen monomers was much higher than the younger specimens. However, the collagen monomers could not be in a fully crosslinked format because they were still soluble in liquid. Hence, the possible architecture of the older collagen monomers was a branched architecture. The alignment experiments under a magnetic field supported this hypothesis. Individual collagen molecules alone are not sufficiently diamagnetically anisotropic to be significantly aligned even in the presence of a very strong magnetic field. However, when the individual molecules come together in an ordered, relatively parallel fashion, to make fibres their anisotropies add together so fibers become aligned when assembled in a sufficiently strong field. This process of alignment worked successfully with 8 week old collagen specimens. However, the older (96 week) collagen did not orient, possibly due to the presence of large branched aggregates, which have a much reduced anisotropy because the molecules are not all in a parallel arrangement. Thus the older collagen specimens did not align under a magnetic field. Interestingly, we were unable to successfully align the youngest collagen from the 2-4 day old rats (babies) under the magnetic field. The possible reason for this may be the gelation programme. The baby collagen solution was the least viscous and took the longest time to gel. However, we used the same heating programme as for 8 week old collagen for the magnetic alignment experiments. The gelation time and programme used may not been suitable for the extremely small collagen monomers. Further investigation is required to clarify the mechanism behind this phenomenon. 
It has been revealed that gelation of collagen is not a one-step process. There are several distinct stages for the fibril assemblies. ${ }^{15}$ The variation in the quality of collagen monomers will alter these stages. The less movable monomers (due to branching or light cross-linking) or high molecular weight molecules resulted in lower density and less organised fibrils, fibres and fibre bundles. This may explain the mechanical measurements in this study. Random cross-linking and lack of organisation during the assembly of collagen fibres in the older collagen led to less flexibility and more fragile constructs, manifesting as a low elastic moduli compared to the younger specimens in the acellular hydrogels. In addition, Scanning Electron Microscope (SEM) studies ${ }^{1}$ on collagen structure in relation to increasing age have revealed that the fibre diameter is more heterogeneous in younger collagen and larger diameter fibres are present. Fibre diameter decreases with increasing age so it is possible that thicker fibres in the younger collagens are stronger than the thinner fibres observed in older collagen, thus resulting in a higher elastic modulus. Kuntz and Saltzmann ${ }^{17}$ have previously reported that fibre diameter is not affected by collagen concentration, demonstrating that a decrease in fibre diameter is not affected by collagen concentration, thus a decrease in fibril diameter must be linked to increasing age.

For the variation of elastic moduli in cell-containing constructs, a slightly different mechanism was involved, which was the cellular contraction capability. In general, fibroblasts contract more in a soft hydrogel construct and contraction ceases when they reach a hard environment. In our study, the fibroblasts contracted the soft, well organised and dispersed collagen fibre bundles in the younger collagen constructs. Thus, high contraction rates have been observed. Whilst in the older collagen specimens, the fibroblasts were in contact with a highly cross-linked network. Locally, the stiffness or hardness of the older collagen environment was much higher compared to the younger counterpart, hence resulting in a lower contraction. The acellular and cellular contraction experiments further support that aged collagen is not really "stiffer" or "stronger". Instead, it is weak and less deformable. Our multiple measurements can be used to predict the microstructural changes in collagen during the ageing process through macroscopic parameter differences.

It has been frequently documented that age-dependent intra- and inter-molecular cross-linking results in changes in the biophysical characteristics of purified soluble collagen. ${ }^{1}$ Formation of AGEs in collagen may be the main process for the cross-linked structure. Accumulation of AGEs in collagenous tissue negatively affects tissue function ${ }^{16}$ as it affects cellular signalling and activation of gene expression. ECM composition, matrix modelling proteinases and integrins are all important factors modulating cell behaviour, in terms of migration, proliferation, spatial organisation and differentiation in vitro and in vivo. ${ }^{1}$

It has been frequently documented that age-dependent intra- and inter-molecular cross-linking results in changes in the biophysical characteristics of purified soluble collagen. ${ }^{1}$ Formation of AGEs might be the main process for the cross-linked structure. Accumulation of AGEs in collagenous tissue negatively affects tissue function ${ }^{15}$ as it affects cellular signalling and activation of gene expression. ECM composition, matrix modelling proteinases and integrins are all important factors modulating cell behaviour, in terms of migration, proliferation, spatial organisation and differentiation in vitro and in vivo. ${ }^{1}$ Our findings are in agreement with previous studies ${ }^{1,6}$ in that increasing age causes structural changes in collagen, which triggers downstream reactions. In studies on intramuscular connective tissue ${ }^{15}$ it was suggested that differences in AGE concentration in young and old tissues in vivo was related to the chronological component of ageing in that the longer the protein residues are exposed to glucose, the greater is the chance of AGEs formation. ${ }^{15}$ The rate by which glycation occurs may be influenced by the location of glycation sites ${ }^{8}$ which differ with age, thus causing increased differences in the collagen structure. Age-related differences in the degree of collagen crosslinking certainly lead to changes in rigidity of 3D structure of the reconstructed collagen gels. ${ }^{1}$

\section{CONCLUSION}

It has been demonstrated that increasing age of collagen has a direct impact on the biomechanical properties of 3D collagen constructs. A combination of non-destructive monitoring techniques has been utilized to demonstrate the effect the collagen matrix has on the cells' ability to manipulate and remodel their surrounding environment. We are able to predict microscopic differences in the collagen monomer and hydrogel by measuring macroscopic parameters, which provides a good platform to further investigate the mechanisms of ageing and the screening of new agents for slowing the ageing process. 


\section{ACKNOWLEDGEMENTS}

The corneal specimens from Birmingham and Midland Eye Centre (UK) are highly appreciated. Funding from the Doctoral Training Centre of Regenerative Medicine, (Engineering and Physical Sciences Research Council, UK), ALLIANCE bilateral programme (EGIDE and British Council) and the exchange programme in EuroMagNET II (Grenoble, France) are gratefully acknowledged. M.G. is a recipient of a fellowship from the Région ChampagneArdenne, France. We thank the CRETA/CNRS laboratory in Grenoble for the use of their magnet and for technical help. Special thanks to KMF Precision Sheet Metal Ltd. (UK) for the kind donation of the specimen clamping apparatus.

\section{REFERENCES}

[1] Jiang, S. T., Liao, K. K., Liao, M.C., Tang, M. J., "Age effect of type I collagen on morphogenesis of Mardin-Darby canine kidney cells," Kidney Int. 57, 1539-1548 (2000).

[2] Willems, N. M. B. K., Langenbach G. E. J., Everts V. et al. "Age-Related Changes in Collagen Properties and Mineralization in Cancellous and Cortical Bone in the Porcine Mandibular Condyle" Calcif Tissue Int. 88. 348-349 (2010)

[3] Schulze, C., Wetzel, F., Kueper, T., et al. "Stiffening of human skin fibroblasts with age," Clin Plast Surg. 39, 9-20. (2012)

[4] Takino, J., Yamagishi, S., Takeuchi, M., "Cancer malignancy is enhanced by glyceraldehyde-derived advanced glycation end-products," Journal of oncology. 2010:739-852 (2010).

[5] Mikulikova, K., Eckhardt, A., Pataridis, S., Miksik, I., "Study of posttranslational non-enzymatic modifications of collagen using capillary electrophoresis/mass spectrometry and high performance liquid chromatography/mass spectrometry," Journal of Chromatography a. 1155, 125-133 (2007).

[6] Bruel, A. and Oxlund, H., "Changes in biomechanical properties, composition of collagen and elastin, and advanced glycation endproducts of the rat aorta in relation to age," Atherosclerosis. 127, 155-165 (1996).

[7] Singh, R., Barden, A., Mori, T., Beilin, L.. "Advanced glycation end-products: a review," Diabetologia. 45, 293-293 (2002).

[8] Lingelbach, L. B., Mitchell, A. E., Rucker, R. B., McDonald, R.B., "Accumulation of advanced glycation endproducts in aging male Fischer 344 rats during long-term feeding of various dietary carbohydrates," J Nutr. 130, 1247-1255 (2000).

[9] Haar, J. L. and Ackerman, G. A., "Phase and Electron Microscopic Study of Vasculogenesis and Erythropoiesis in Yolk Sac of Mouse," Anat Rec. 170, 199 (1971).

[10] Garnotel, R., Rittié, L., Poitevin, S., et al., "Human Blood Monocytes Interact with Type I Collagen Through $\alpha \times ß 2$ Integrin (CD11c-CD18, gp150-95)," The Journal of Immunology. 164, 5928-5934 (2000).

[11] Millerot-Serrurot, E., Guilbert, M., Fourre, N., et al., "3D collagen type I matrix inhibits the antimigratory effect of doxorubicin," Cancer Cell Int. 10, 26 (2010).

[12] Ahearne, M., Liu, K., El Haj, A. J., Then, K. Y., Rauz, S., Yang, Y., "Online Monitoring of the Mechanical Behavior of Collagen Hydrogels: Influence of Corneal Fibroblasts on Elastic Modulus," Tissue Eng Part C-Methods. 16, 319-327 (2010). 
[13] Liu,K. K. and Ju, B. F., "A novel technique for mechanical characterization of thin elastomeric membrane," J Phys D-Appl Phys. 34, $91-94$ (2001).

[14] Yang, Y., Bagnaninchi, P. O., Ahearne, M., Wang, R. K., Liu, K., "A novel optical coherence tomography-based micro-indentation technique for mechanical characterization of hydrogels," J R Soc Interface. 4,1169-1173 (2007).

[15] Trelstad, R. L., Hayashi, K., Gross, J. "Collagen fibrillogenesis: intermediate aggregates and suprafibrillar order," Proc Natl Acad Sci U S A. 73, 4027-4031 (1976).

[16] Haus, J. M., Carrithers J. A., Trappe, S. W., Trappe, T. A., "Collagen, cross-linking, and advanced glycation end products in aging human skeletal muscle," J Appl Physiol. 103, 2068-2076 (2007).

[17] Kuntz, R.M. and Saltzman, W. M. "Neutrophil motility in extracellular matrix gels: Mesh size and adhesion affect speed of migration," Biophys J. 72, 1472-1480 (1997). 